\title{
Northrop Frye
}

\author{
14 Iuglio 1912-23 febbraio 1991
}

È con un articolo di Frye sul Cortegiano che si apriva nel 1980 il primo numero di Quaderni d'italianistica. La disponibilità dello studioso e i suoi incoraggiamenti per progetti culturali canadesi nell'arte, nella letteratura e nella critica non hanno bisogno di essere ricordati, ma nel caso specifico c'erano almeno due ragioni in più a sostenerli: il mito dell'Italia, alma mater della cultura occidentale moderna, visitata da lui più volte con lo stesso spirito di Milton trecent'anni prima, e poi un rammarico tutto personale per non aver mai studiato sistematicamente (sono parole sue) la cultura italiana del tardo Medioevo e del primo Rinascimento. Non rientrava certo Dante in questa dichiarata carenza se la Divina Commedia è l'opera letteraria più citata nel suo ultimo libro, Words with Power, e se passi del Convivio e della lettera a Cangrande sono fondamentali nella sua teoria del simbolo già in Anatomy of Criticism. Ricordo di aver accompagnato anni fa da Frye Romano Luperini che gli consegnò una copia della sua rivista, L'ombra d'Argo. "Ah, Paradiso 33", disse appena l'ebbe vista. Amava l'arte romanica e gotica. Spesso nei suoi scritti ricordava le Madonne di Siena e di Torcello assimilandone la spiritualità e la nettezza del disegno alle incisioni di Blake. Gli affreschi di Giotto erano per lui tra le espressioni più alte di civiltà di tutti i tempi, civiltà intesa come attuazione del potenziale creativo dell'immaginazione umana. La divinità è nell'uomo, insegnava, nella consapevolezza di questo suo potere illimitato. Credeva nella trascendenza, ma si considerava parte di una tradizione "eretica" di fronte alla Bibbia da leggere senza schemi concettuali o dottrinari inibitori. Una sua interpretazione della creazione di Adamo nella Cappella Sistina serve più di un lungo discorso per chiarire la fede nell'uomo di Frye: "Ho visto sempre l'affresco di Michelangelo in modo rovesciato: non è un essere astratto e distante che crea l'uomo, è l'uomo che con la sua immaginazione raggiunge la coscienza della divinità, crea Dio". Ed ecco, allora, perché per Frye si parte sempre dall'uomo, dai suoi bisogni esistenziali, che sono di sopravvivenza e di riconoscimento della propria identità umana e divina allo stesso tempo. La letteratura, dice in Words with Power, è una tecnica di meditazione. L'oggetto della meditazione è la ricerca di sé stessi individualmente e come parte della collettività umana, è l'oracolo di Delfi. I poeti sono "children of concern" perché in essi i bisogni primari sono i bisogni primari dell'uomo e nella poesia, al centro della letteratura che è al centro delle arti, sta il germe che trasforma "humanity in the flower of humanity".

FRANCESCO GUARDIANI 